\section{From phenotype to genotype}

Phenotypic mutations result from errors in transcription or translation that lead to the production of protein variants from unmodified genes; however, the role of this process in evolution has been unclear. Now, a study on the evolution of protein paralogues provides the first direct evidence that phenotypic mutations paved the way for genetic adaptation through gene duplication.

Yanagida et al. focused on the Saccharomyces cerevisiae Idp2 and Idp3 proteins, which compartmentalize to the cytosol and the peroxisome, respectively, and diverged following the yeast whole-genome duplication (WGD) event. To investigate the compartmentalization of Idp3, the authors searched for a carboxy-terminal peroxisomal targeting sequence, called PTS1, in S. cerevisiae-related species that diverged before or after the WGD. Both pre-WGD and post-WGD species contained an IDP2 gene encoding a cytosolic isoform, but only post-WGD strains contained an IDP3 paralogue with a PTS1 signature for peroxisomal targeting. In complementation experiments, only IDP2 that was modified to contain PTS1 could complement an IDP3 mutation. This suggested that, following the WGD, Idp2 remained a cytosolic protein and Idp3 acquired a PTS1 signature, causing it to localize to the peroxisome.

Interestingly, the authors identified putative PTS1 motifs that were +1 nucleotide out of reading frame, downstream of pre-duplication IDP2 genes in several Saccharomycetaceae species. Unveiling these cryptic motifs through the introduction of a single-nucleotide deletion resulted in peroxisomal targeting of the product, suggesting that transcriptional or translational frameshift errors in an ancestral IDP2 gene might result in expression of a peroxisomal isoform, in addition to the usual cytosolic isoform. Indeed, mass spectrometry and fluorescent cell imaging confirmed the coexistence of cytosolic and peroxisomal isoforms of Idp2 in a pre-WGD strain containing only an IDP2 gene with a cryptic PTS1.

Exploring the mechanism of cryptic PTS1 recruitment, the authors examined a homonucleotide repeat situated just upstream of the IDP2 stop codon of one pre-WGD strain. The introduction of silent mutations here abolished PTS1 functionality, confirming this as the site of phenotypic mutation. Furthermore, RT-PCR analysis revealed the preservation of this homonucleotide repeat at the RNA level, suggesting that production of peroxisomal Idp protein from ancestral IDP2 is due to translational, not transcriptional, frameshifting. Remarkably, laboratory evolution experiments indicated that the phenotypic mutations that led to peroxisomal targeting of Idp2 protein became fixed in post-duplication IDP 3 by genetic deletions occurring at the very same site.

These experiments show that $S$. cerevisiae IDP3 diverged from a pre-duplication IDP2 gene that already had the capacity for peroxisomal compartmentalization through phenotypic mutation. Therefore, gene duplication did not initiate but rather followed the phenotypic divergence of compartmentalization. These findings suggest that genotypes favouring phenotypic variability enhance the potential for future evolutionary genetic adaptations.

\section{Denise Waldron}

ORIGINAL RESEARCH PAPER Yanagida, H. et al. The evolutionary potential of phenotypic mutations. PLoS Genet. 11, e1005445 (2015)

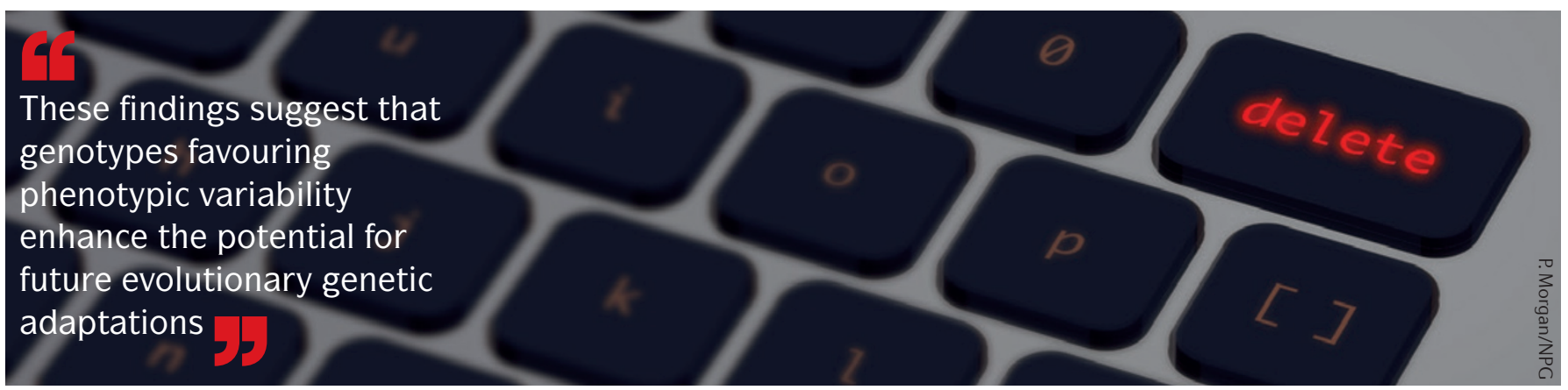

